Commun. math. Phys. 13, 1-23 (1969)

\title{
Fields, Observables and Gauge Transformations I
}

\author{
Sergio Dopltcher * \\ Faculté des Sciences, Université d'Aix-Marseille
}

Rudolf HaAG and John E. Roberts

II. Institut für Theoretische Physik der Universität Hamburg

Received April 14, 1969

\begin{abstract}
Starting from an algebra of fields $\mathfrak{F}$ and a compact gauge group of the first kind $\mathscr{G}$, the observable algebra $\mathfrak{A}$ is defined as the gauge invariant part of $\mathfrak{F}$. A gauge group of the first kind is shown to be automatically compact if the scattering states are complete and the mass and spin multiplets have finite multiplicity. Under reasonable assumptions about the structure of $\mathfrak{F}$ it is shown that the inequivalent irreducible representations of $\mathfrak{A}$ ("sectors") which occur are in one-to-one correspondence with the inequivalent irreducible representations of $\mathscr{G}$ and that all of them are "strongly locally equivalent". An irreducible representation of $\mathfrak{A}$ satisfies the duality property only if the sector corresponds to a 1-dimensional representation of $\mathscr{G}$. If $\mathscr{G}$ is Abelian the sectors are connected to each other by localized automorphisms.
\end{abstract}

\section{Introduction}

One of the most characteristic assumptions of relativistic Quantum Field Theory is the principle of locality. In its most general form it may be stated as follows:

There is a correspondence between regions in space-time and algebras of observables

$$
\mathcal{O} \rightarrow \mathfrak{Q}(\mathcal{O})
$$

Here and throughout this paper $\mathcal{O}$ denotes an open and finitely extended region of space-time; $\mathfrak{A}(\mathcal{O})$ is the algebra generated by all the observables which can be measured within $\mathcal{O}$. If $\mathcal{O}_{1}$ and $\mathcal{O}_{2}$ are two regions which lie totally spacelike with respect to each other, $\mathfrak{A}\left(\mathcal{O}_{1}\right)$ and $\mathfrak{A}\left(\mathcal{O}_{2}\right)$ are required to commute. The argument for this is based on Einstein's relativistic causality principle which states that no physical influence is possible between the two regions. Hence a measurement in $\mathcal{O}_{1}$ should not disturb a measurement in $\mathrm{O}_{2}$.

Historically, in the development of Quantum Field Theory, it has been found necessary (or at least convenient) to introduce unobservable "local" quantities (e.g. "charged fields", Fermi-Dirac fields etc.). Such quantities, being unobservable as a matter of principle, need not commute

^ On leave of absence from Instituto di Fisica G. Marconi, Università di Roma.

1 Commun.math.Phys., Vol.13 
at space-like distances. Indeed to obtain Fermi statistics for certain particle types, JORDAN and WIGNER postulated the anticommutativity of certain unobservable fields at space-like distances. In recent years more complicated commutation relations have also been discussed ("para-fields").

In all such cases we have an assignment

$$
\mathcal{O} \rightarrow \mathfrak{F}(\mathcal{O})
$$

where the "field algebra" $\mathfrak{F}(\mathcal{O})$ of the region $\mathcal{O}$ is larger than "observable algebra" $\mathfrak{A}(\mathcal{O})$. We know on general grounds [1] that the algebraic structure of the observable algebra should contain all physical information about the system. In particular this means that the existence of particles not obeying Bose statistics or of particles carrying charge quantum numbers must already be expressed by certain structural properties of the observable algebra $\mathfrak{A}$, although $\mathfrak{A}$ does not contain any elements which anticommute with their spatial translates at large distances or elements which transfer charges. In other words, it should be possible to construct the "field algebra" $F$ (uniquely to the degree to which $\mathfrak{F}$ is physically relevant) from a given observable algebra $\mathfrak{A}$. Such a construction has been attempted by Borchers [2]. This interesting attempt unfortunately uses certain structural properties of the representations of $\mathfrak{A}$ absent in typical cases ${ }^{1}$. Our present paper is, therefore, devoted entirely to the opposite problem: given the representation of the field algebra generated from the vacuum state, find the properties of the irreducible representations of the observable algebra which are contained in it. The more interesting problem of constructing the field algebra from $\mathfrak{A}$ will be tackled in a subsequent paper.

The main assumptions we make are standard and will be listed here:

1. We consider a Hilbert space $\mathscr{H}$ and, acting on it, the algebra of all bounded operators $\mathscr{B}(\mathscr{H})$. Associated with each finitely-extended, open space-time region $\mathcal{O}$ there is a $*_{\text {-subalgebra }} \mathfrak{F}(\mathcal{O}) \subset \mathscr{B}(\mathscr{H})$. Without loss of generality in any of the following arguments, we can assume that $\mathfrak{F}(\mathcal{O})$ is weakly closed, i.e.

$$
\mathfrak{F}(\mathcal{O})^{-}=\mathfrak{F}(\mathcal{O})^{\prime \prime}=\mathfrak{F}(\mathcal{O}) .
$$

Here $\mathscr{S}$ - denotes the weak closure of a set $\mathscr{S}$ of bounded operators; $\mathscr{S}^{\prime}$ denotes the commutant of $\mathscr{S}$. The correspondences (1.1), (1.2) have to satisfy "isotony" i.e. $\mathcal{O}_{1} \supset \mathcal{O}_{2}$ implies $\mathfrak{A}\left(\mathcal{O}_{1}\right) \supset \mathfrak{A}\left(\mathcal{O}_{2}\right)$ and $\mathfrak{F}\left(\mathcal{O}_{1}\right) \supset \mathfrak{F}\left(\mathcal{O}_{2}\right)$.

The total field algebra $\mathfrak{F}$ is defined as the closure in the norm topology of the union of all $\mathfrak{F}(\mathcal{O})$ :

$$
\mathfrak{F}=\overline{\cup \mathfrak{F}(\mathcal{O})} .
$$

1 These properties were suggested by Theorem 13 of [3] the proof of which contains an error. 
The Hilbert space is irreducible with respect to $\mathfrak{F}$, i.e.

$$
\mathfrak{F}^{-}=\mathfrak{F}^{\prime \prime}=\mathscr{B}(\mathscr{H}) \text {. }
$$

2. We have a strongly continuous unitary representation $U(L)$ of the Poincaré group $\mathscr{P}_{+}^{\uparrow}$ in $\mathscr{H}$. Here $L$ is a general element of $\mathscr{P} \uparrow$ and $U(L)$ its representative. ${ }^{1 \text { a }}$

The generators of the space-time translations are called the energymomentum operators. They satisfy the spectrum condition: there is exactly one vector $\Omega$ (the vacuum state) which belongs to the eigenvalue zero of the energy operator. The energy operator $P_{0}$ has its spectrum confined to the range $E \geqq 0$. This representation induces automorphisms of $\mathfrak{F}$ denoted by $\alpha_{L}$ :

$$
U(L) F U(L)^{-1}=\alpha_{L}(F) .
$$

$\alpha_{L}$ transforms a subalgebra $\mathfrak{F}(\mathcal{O})$ into the subalgebra of the transformed region $\mathfrak{F}(L \mathcal{O})$.

3. There is a compact group $\mathscr{G}$, the gauge group, and a faithful, strongly continuous unitary representation of it $g \in \mathscr{G} \rightarrow U(g) \in \mathscr{B}(\mathscr{H})$ which induces a group of automorphisms of $\mathfrak{F}$ :

$$
U(g) F U(g)^{-1}=\alpha_{g}(F) .
$$

The representations $U(L)$ and $U(g)$ commute, i.e. $U\left(\mathscr{P}_{+}^{\uparrow}\right) \subset U(\mathscr{G})^{\prime}$. Also, $\alpha_{g}$ acts in a strictly local way on $\mathfrak{F}$, i.e.

$$
\alpha_{g}(\mathfrak{F}(\mathcal{O}))=\mathfrak{F}(\mathcal{O}) \text { for all } \mathcal{O} \text {. }
$$

4. The observable algebra of the region $\mathcal{O}$ is defined as the subset of $\mathfrak{F}(\mathcal{O})$ which is invariant under gauge transformations:

$$
\mathfrak{A}(\mathcal{O})=\mathfrak{F}(\mathcal{O}) \cap U(\mathscr{G})^{\prime} .
$$

The total observable algebra is defined analogously to (1.4) as

$$
\mathfrak{A}=\overline{\cup \mathfrak{U}(\mathscr{O})} \text {. }
$$

The observable algebra shall have the local commutation relations

$$
\mathfrak{A}\left(\mathcal{O}_{1}\right) \subset \mathfrak{A}\left(\mathcal{O}_{2}\right)^{\prime} \text { if } \mathcal{O}_{1} \text { is space-like to } \mathcal{O}_{2} \text {. }
$$

The structure specified above has been tailored to describe gauge invariance of the first kind. From a physical point of view, one expects it to be directly relevant only in the absence of long-range forces, e.g. if one wants to describe charge quantum numbers in strong interaction physics. The significant assumption - which fails for instance in Quantum Electrodynamics - is the covariance of the field algebra under the Poincaré group (in a Hilbert space with positive metric). Related to this is the requirement that gauge transformations and Poincaré trans-

1 a In this paper $\mathscr{P} \uparrow$ will always be understood to be the covering group of the inhomogeneous Lorentz group. 
formations commute, and this excludes, of course, gauge groups of the second kind. On the other hand, in a theory with only short range forces, the assumptions are coherent. In particular, if we omit the topological requirements on the gauge group [compactness and strong continuity of $g \rightarrow U(g)]$ in 3 , we can consider the group $\mathscr{G}_{\max }$ consisting of all unitary operators $U$ on $\mathscr{H}$ satisfying:

$$
\begin{aligned}
{[U, U(L)] } & =0, \quad L \in \mathscr{P} \uparrow, \\
U \mathscr{F}(\mathcal{O}) U^{-1} & =\mathfrak{F}(\mathcal{O}), \quad \text { for all } \mathcal{O}, \\
U \Omega & =\Omega .
\end{aligned}
$$

We equip this group with the strong operator topology. Then we show in section II that $\mathscr{G}_{\max }$ is compact if the theory has a complete particle interpretation, with the usual definition of scattering states, and if no particle multiplet has infinite multiplicity. The gauge group is a subgroup of $\mathscr{G}_{\max }$ and we can take it to be a closed subgroup without affecting the definition of the observable algebra. Thus $\mathscr{G}$ will be compact and $g \rightarrow U(g)$ strongly continuous.

One usually considers gauge groups of the first kind to be groups generated by one or more independent charges. In this case, $\mathscr{G}$ is an Abelian group, the direct product of circle groups (phase factors). However, if one considers strong interaction physics by itself, the natural gauge group is the isospin group [isomorphic to $S U(2)]$. One of the aims of this investigation is to analyse the effect of a non-Abelian gauge group on the structure of the family of representations of $\mathfrak{A}$. Section IV shows how an essential distinction between the Abelian and the non-Abelian case can be made in terms of duality. A certain duality requirement on the field algebra [see Eq. (1.23)] implies that the irreducible representation $\pi$ of the observable algebra corresponding to a specific sector satisfies the duality relation

$$
\pi\left(\mathfrak{U}\left(\mathcal{O}^{\prime}\right)\right)^{-}=\pi(\mathfrak{A}(\mathcal{O}))^{\prime}
$$

if the sector corresponds to a one-dimensional representation of the gauge group. In (1.15) as in the remainder of this paper, $\mathfrak{A}\left(\mathcal{O}^{\prime}\right)$ denotes the $C^{*}$-algebra generated by all $\mathfrak{A}\left(\mathcal{O}_{i}\right)$ with $\mathcal{O}_{i}$ totally space-like to $\mathcal{O}$. The relation (1.15) will not hold in sectors associated with the irreducible representations of the gauge group of dimension greater than one which occur if $\mathscr{G}$ is not Abelian.

The relationship between the irreducible representations of $\mathfrak{A}$ contained in $\mathscr{H}$ and the irreducible representations of $\mathscr{G}$ is studied in section III. According to the standard terminology, an element of the "spectrum" of a group (or algebra) means an equivalence class of irreducible representations. For convenience, we refer to the set of equivalence classes of irreducible representations of $\mathfrak{A}$ contained in $\mathscr{H}$ as the "physical spectrum" 
of $\mathfrak{A}$. The main conclusion of section III is that the physical spectrum of $\mathfrak{A}$ is in natural $1-1$ correspondence with the complete spectrum of $\mathscr{G}$. For this result, the following two additional assumptions are made:

5. The "cluster property" of the vacuum state vector $\Omega^{2}$ :

$$
\lim _{|\boldsymbol{x}| \rightarrow \infty}\left\langle\Omega, F_{1} \alpha_{\boldsymbol{x}}\left(F_{2}\right) F_{3} \Omega\right\rangle=\left\langle\Omega, F_{1} F_{3} \Omega\right\rangle\left\langle\Omega, F_{2} \Omega\right\rangle
$$

for all $F_{1}, F_{2}, F_{3} \in \mathscr{F}$. Of course, $\boldsymbol{x} \in \mathscr{P} \uparrow$ denotes a spatial translation.

6 . The Reeh-Schlieder property of analytic vectors:

If $\Psi$ is an analytic vector for the energy operator (a.f.e.-vector) ${ }^{3}$, it is cyclic and separating for each $\mathfrak{F}(\mathcal{O})$ i.e. $\mathfrak{F}(\mathcal{O}) \Psi$ is dense in $\mathscr{H}$ and $F \Psi=0, F \in \mathscr{F}(\mathcal{O})$ implies $F=0$.

As Borchers has shown [4], assumption 6 is equivalent to "weak additivity" in the following sense

$$
\begin{aligned}
& \bigvee_{x \in \boldsymbol{R}^{4}} \mathfrak{F}(\mathcal{O}+x)=\mathscr{B}(\mathscr{H}),{ }^{4} \\
& \bigvee_{x \in \boldsymbol{R}^{4}} \mathfrak{F}(\mathcal{O}+x)^{\prime}=\mathscr{B}(\mathscr{H}) .
\end{aligned}
$$

This should hold if the scheme bears any resemblance to field theory.

In Sections V and VI, we investigate various forms of local equivalence for the representations of the observable algebra in different sectors (compare [2], [3]). "Local equivalence" means that the restrictions of the representations to each local subalgebra $\mathfrak{A}(\mathcal{O})$ are unitarily equivalent. "Strong local equivalence" means that the same is true for the restrictions to each $\mathfrak{A}\left(\mathcal{O}^{\prime}\right)$. An even stronger form of local equivalence could be defined by requiring that the representations of the "relative commutants" $\mathfrak{A}(\mathcal{O})$ of the local algebras are unitarily equivalent. Here $\mathfrak{A}^{c}(\mathcal{O})$ is the $C^{*}$-subalgebra of $\mathfrak{A}$ consisting of all elements of $\mathfrak{A}$ which commute with every element of $\mathfrak{A}(\mathcal{O})$. Considered as an operator algebra on $\mathscr{H}, \mathfrak{A}^{c}(\mathcal{O})$ is given by

$$
\mathfrak{A}^{c}(\mathcal{O})=\mathfrak{A}(\mathcal{O})^{\prime} \cap \mathfrak{A} .
$$

Our conclusion is that typically the representations of $\mathfrak{A}^{c}(\mathcal{O})$ in the different sectors are disjoint (Section V) but that the different sectors are strongly locally equivalent (Section VI). The strong local equivalence follows from a mild restrictive assumption on the commutation structure of $\mathfrak{F}$ :

${ }^{2}$ If $\mathfrak{F}$ has Bose or Fermi commutation structure, then (1.16) is a consequence of the earlier assumptions.

${ }^{3} \Psi$ is an a.f.e.-vector if the power series $\sum_{n} \frac{\tau^{n}}{n !}\left\|P_{0}^{n} \Psi\right\|$ has a non-zero radius of convergence in $\tau$.

${ }^{4}$ The symbol $V$ is used to denote the least upper bound in the lattice of von Neumann algebras on $\mathscr{H}$ ordered under inclusion. 
7. The field algebra in $\mathcal{O}$ commutes with the observable algebra in $\mathcal{O}^{\prime}$

$$
\mathfrak{F}(\mathcal{O}) \subset \mathfrak{A}\left(\mathcal{O}^{\prime}\right)^{\prime}
$$

The disjointness result on the other hand is derived with the help of assumption 8, formulated in Section $V$, whose physical content is roughly that there exist local currents generating the gauge transformations. We illustrate this connexion here with a heuristic argument for the case of the free charged scalar field. Let $\mathcal{O}_{R}=\left\{x:\left|x_{0}\right|+|\boldsymbol{x}|<R\right\}$, the open double cone of "radius" $R$ centred on the origin. Pick $R-\delta$ such that $\mathcal{O} \subset \mathcal{O}_{R-\delta}$ and let $j_{0}(x)$ be the charge density, $f_{R}$ a smooth function of $\boldsymbol{x} \in \boldsymbol{R}^{3}$, vanishing for $|\boldsymbol{x}|>R$ and equal to 1 for $|\boldsymbol{x}|<R-\delta$, and $g_{\delta}$ a smooth function of $x_{0}$ with total integral 1, vanishing for $\left|x_{0}\right|>\delta$. The local charge $j_{0}\left(f_{R} g_{\delta}\right)$ is associated with $\mathfrak{H}^{c}(\mathcal{O})$ and converges in a certain sense on each sector to the corresponding charge quantum number as $R \rightarrow \infty$. On the other hand it should always tend to the same $c$-number on all subspaces yielding mutually equivalent representations for $\mathfrak{A}^{c}(\mathcal{O})$.

We turn now to the question of the commutation structure of $\mathfrak{F}$. One may note that the way in which the observable algebra is defined [see Eq. (1.9)] imposes certain restrictions through the locality requirement on $\mathfrak{A}$. As $\mathscr{G} \subset \mathscr{G}_{\max }$, we have

where

$$
\mathfrak{A} \supset \mathfrak{A}_{\min }
$$

$$
\mathfrak{A}_{\min }(\mathcal{O})=\mathfrak{F}(\mathcal{O}) \cap U\left(\mathscr{G}_{\max }\right)^{\prime} .
$$

Thus the algebra $\mathfrak{A}_{\min }$, which is determined entirely by $\mathfrak{F}$ and $U(\mathscr{P} \uparrow)$ has to be local. We shall not attempt to classify the possible commutation structures of $\mathfrak{F}$ from this point of view. Instead we consider explicit restrictions on the commutation structure such as assumption 7 above and the "twisted locality" introduced in Section IV.

Assumption 7 may be strengthened to a duality relation used in Section IV:

$$
\mathfrak{A}\left(\mathcal{O}^{\prime}\right)^{-}=\mathfrak{F}(\mathcal{O})^{\prime} \cap U(\mathscr{G})^{\prime}
$$

For Abelian gauge groups, this leads to a very simple description of the relation between the different sectors in terms of "localized automorphisms" of $\mathfrak{A}$ (Section VI). This structure will be the starting point of a subsequent paper on the reconstruction of the field algebra. It will be shown there that it leads to an Abelian gauge group and a field algebra whose commutation properties satisfy the Bose-Fermi alternative.

\section{Compactness of the Gauge Group}

In the introduction, we defined the "maximal gauge group" $\mathscr{G}_{\max }$ which can be associated with a given field algebra. This group is the group of unitary operators on $\mathscr{H}$ satisfying (1.12), (1.13) and (1.14). We 
want to show that it is compact in the strong topology of $\mathscr{H}$ under reasonable physical assumptions.

We consider the subspace $\mathscr{H}^{(1)}$ spanned by the single particle states of $\mathscr{H}$. The restriction of the representation of the Poincare group to $\mathscr{H}^{(1)}$, denoted by $U^{(1)}$, is a direct sum of factor representations:

$$
\begin{aligned}
\mathscr{H}^{(1)} & =\bigoplus_{i}\left(\mathscr{H}_{i} \otimes \mathscr{H}_{i}^{\prime}\right) \\
U^{(1)}(L) & =\bigoplus_{i}\left(U_{\left[m_{i}, s_{i}\right]}(L) \otimes I_{i}^{\prime}\right) .
\end{aligned}
$$

Here the index $i$ denotes a specific value of the mass and spin of a particle multiplet. $U_{\left[m_{i}, s_{i}\right]}$ is the corresponding irreducible representation of $\mathscr{P} \uparrow$ and $\mathscr{H}_{i}^{\prime}$ allows us to distinguish the different particles of the mass and spin multiplet.

We shall now assume that the formalism of collision theory which has been derived in the case of Bose- and Fermi-fields (see e.g. [5]) can be applied analogously in our context. Let $\mathfrak{F}_{\infty}$ denote the (norm dense) *-subalgebra of $\mathfrak{F}$ consisting of those elements which are quasilocal of infinite order ${ }^{5}$. We can find a collection $B_{i, \lambda}$ of operators from $\mathfrak{F}_{\infty}$ such that

$$
B_{i, \lambda} \Omega=\phi_{i, \lambda} \in \mathscr{H}_{i} \otimes \mathscr{H}_{i}^{\prime}
$$

and such that the whole single particle space $\mathscr{H}^{(1)}$ is spanned by the spatial translates of the vectors $\phi_{i, \lambda}$. We define, in the usual way (see e.g. [5]), time-dependent creation operators

$$
B_{i, \lambda}^{f_{i}}(t)=\int_{x_{0}=t} f_{i}(x) \alpha_{x}\left(B_{i, \lambda}\right) d^{3} x
$$

where $f_{i}(x)$ is a function whose Fourier transform $\tilde{f}_{i}(p)$ has support concentrated on the positive shell of the hyperboloid of mass $m_{i}$ :

$$
\tilde{f}_{i}(p)=\phi(\boldsymbol{p}) \delta\left(\boldsymbol{p}^{2}+m^{2}-p_{0}^{2}\right) \theta\left(p_{0}\right)
$$

and $\phi(\boldsymbol{p})$ is infinitely often differentiable and of compact support. The vector $B_{i, \lambda}^{f_{i}}(t) \Omega$ is then independent of $t$ and lies in $\mathscr{H}_{i} \otimes \mathscr{H}_{i}^{\prime}$. We assume that the states corresponding to configurations of several incoming particles can be constructed with the help of the operators (2.4) in the usual fashion, i.e. (omitting for brevity the indices $i, \lambda$ ) that the state vectors

$$
\lim _{t \rightarrow-\infty} B^{f_{i}}(t) \ldots B^{f_{n}}(t) \Omega \equiv\left(\phi_{1} \times \phi_{2} \times \ldots \times \phi_{n}\right)^{i n}=\Phi
$$

exist as norm limits and depend only on the $\phi_{i}=B_{f_{i}}(t) \Omega$. The order of

${ }^{5}$ Let $\mathcal{O}_{n}$ be a sequence of concentric double cones with base radii $R_{n}$ tending to infinity for $n \rightarrow \infty$ and let $d_{n}$ be the norm distance between $F \in \mathfrak{F}$ and the set $\mathfrak{F}\left(\mathcal{O}_{n}\right)$. Then $F$ is called quasilocal of order $N$ if

$$
\lim _{n \rightarrow \infty} R_{n}^{k} d_{n}=0 \quad \text { for } \quad 0 \leqq k \leqq N .
$$


the factors is, of course, relevant if we are dealing with particles other than Bosons. The mapping

$$
\phi_{1} \otimes \phi_{2} \otimes \ldots \otimes \phi_{n} \rightarrow\left(\phi_{1} \times \phi_{2} \times \ldots \times \phi_{n}\right)^{i n}
$$

is assumed to be a continuous linear mapping of $\bigotimes_{i=1}^{n}\left(\mathscr{H}_{i} \otimes \mathscr{H}_{i}^{\prime}\right)$ into $\mathscr{H}$.

In addition to these features of collision theory which have been established in the Bose-Fermi case we assume

(i) The linear span of the ranges of all the maps (2.7) is dense in $\mathscr{H}$ (i.e. the incoming states form a complete set).

(ii) Each particle multiplet has only a finite number of components, i.e. each $\mathscr{H}_{i}^{\prime}$ is finite dimensional.

Let us now consider the group $\mathscr{G}_{\infty}$ of all unitary operators which commute with the Poincaré transformations, act as the identity on the vacuum vector and transform $\mathfrak{F}_{\infty}$ into itself. The first two conditions defining $\mathscr{G}_{\infty}$ are the same as conditions (1.12) and (1.14) on the elements of $\mathscr{G}_{\max }$. The last condition

$$
U \mathfrak{F}_{\infty} U^{-1}=\mathfrak{F}_{\infty}
$$

is weaker than the corresponding condition (1.13). $\mathscr{G}_{\max }$ is thus a subgroup of $\mathscr{G}_{\infty}$. By (1.12), $\mathscr{G}_{\infty}$ transforms the single particle subspace $\mathscr{H}^{(1)}$ into itself. We denote its restriction to $\mathscr{H}^{(1)}$ by $\mathscr{G}_{\infty}^{(1)}$. Considering $\mathscr{G}_{\infty}$ and $\mathscr{G}_{\infty}^{(1)}$ as topological groups with the strong operator topologies of $\mathscr{H}$ and $\mathscr{H}^{(1)}$ respectively, we first note that $\mathscr{G}_{\infty}$ and $\mathscr{G}_{\infty}^{(1)}$ are isomorphic. Indeed, (2.6), (2.4) and the properties of a $U \in \mathscr{G}_{\infty}$ imply

$$
U\left(\phi_{1} \times \phi_{2} \times \ldots \times \phi_{n}\right)^{i n}=\left(U \phi_{1} \times U \phi_{2} \times \ldots \times U \phi_{n}\right)^{i n} .
$$

By assumption (i), Eq. (2.9) implies that $U \in \mathscr{G}_{\infty}$ is uniquely determined by its restriction to $\mathscr{G}_{\infty}^{(1)}$ and further, with the continuity of the map (2.7), that the elements of $\mathscr{G}_{\infty}^{(1)}$ are in continuous $1-1$ correspondence with their extensions to $\mathscr{G}_{\infty}$.

Next, let $\mathscr{U}\left(\mathscr{H}_{i}^{\prime}\right)$ be the group of all unitary operators on $\mathscr{H}_{i}^{\prime}$ with the strong operator topology. Since $\mathscr{H}_{i}^{\prime}$ is finite dimensional, this group is compact. The group of all unitaries on $\mathscr{H}^{(1)}$ satisfying (1.12) (equipped with the strong operator topology) is isomorphic to $\prod_{i} \mathscr{U}\left(\mathscr{H}_{i}^{\prime}\right)$, the topological product group of the $\mathscr{U}\left(\mathscr{H}_{i}^{\prime}\right)$. By TychonofF's Theorem $[6 ; \mathrm{I}$, $\S 9$ No 5], this group is again compact. It obviously contains $\mathscr{G}_{\infty}^{(1)}$.

Finally we note that $\mathscr{G}_{\max }$ (though perhaps not $\mathscr{G}_{\infty}$ ) is closed since the three defining relations (1.12), (1.13) and (1.14) remain valid for limits in the strong operator topology.

Putting the three remarks together we have

2.1 Theorem. $\mathscr{G}_{\infty}$ is isomorphic to a subgroup of the compact group $\prod_{i} \mathscr{U}\left(\mathscr{H}_{i}^{\prime}\right) . \mathscr{G}_{\max }$ is a compact subgroup of $\mathscr{G}_{\infty}$. 
Note that it has not been necessary to assume that the total number of different multiplets is finite. In fact, the proof remains valid for a non-separable $\mathscr{H}$ with an uncountable number of multiplets.

\section{The Decomposition into Sectors}

Having seen in detail how asymptotic completeness and the finiteness of particle multiplets restrict the possible nature of the gauge group, and in particular allow us to assume it compact, we turn to the main problem of describing the given structure in terms of representations of the observable algebra and its local subalgebras. The simplest problem we can pose ourselves is that of decomposing the given representation of the observable algebra into its irreducible components. As a preliminary, we must describe how the observable algebra may be obtained from the field algebra by averaging over the gauge group. We begin with two simple lemmas establishing the basic properties of taking means of operators over a compact group.

3.1. Lemma. If $F \in \mathscr{B}(\mathscr{H})$ then $m(F)=\int_{\mathscr{G}} \alpha_{g}(F) d \mu(g)$, where the integral is considered in the weak operator topology and $\mu$ denotes normalized Haar measure. Further

a) $\alpha_{g} \circ m=m \circ \alpha_{g}=m$, i.e. $m$ projects from $\mathscr{B}(\mathscr{H})$ onto $U(\mathscr{G})^{\prime}$.

b) If $F \geqq 0, m(F) \geqq 0$ and $m(I)=I$, i.e. $m$ is a normalized positive mapping.

c) $m \circ m=m$, i.e. $m$ is idempotent.

d) $m$ is ultraweakly continuous; equivalently $m$ is normal, or weakly continuous on the unit ball of $\mathscr{B}(\mathscr{H})$.

Proof. The integral exists as $g \rightarrow U(g)$ is strongly continuous. A compact group is unimodular so Haar measure is left and right invariant, hence a). The positive cone of $\mathscr{B}(\mathscr{H})$ is weakly closed and $\mu$ is normalized hence b). c) follows directly from a). We may prove d) by showing directly that $m$ is continuous on the unit ball of $\mathscr{B}(\mathscr{H})$ using the compactness of $\mathscr{G}$.

Alternatively, we may note that

1. the set of trace class operators $(\tau c)$ is the ultraweak dual of $\mathscr{B}(\mathscr{H})$ $[7 ;$ p. $37-42]$;

2. $m$ maps $(\tau c)$ into itself, in fact $\operatorname{Tr} m(T)=\operatorname{Tr} T$;

3. the map $m:(\tau c) \rightarrow(\tau c)$ is the dual map of $m: \mathscr{B}(\mathscr{H}) \rightarrow \mathscr{B}(\mathscr{H})$, i.e. $\operatorname{Tr}(T m(F))=\operatorname{Tr}(m(T) F), T \in(\tau c), F \in \mathscr{B}(\mathscr{H})$.

3.2. Lemma. Let $\mathfrak{Z}$ be a $C^{*}$-algebra on $\mathscr{H}$ such that $m(\mathfrak{B}) \subset \mathfrak{B}$, then $\left(\mathfrak{Z} \cap U(\mathscr{G})^{\prime}\right)^{-}=\mathfrak{B}^{-} \cap U(\mathscr{G})^{\prime}$.

Proof. Since $m(\mathfrak{B}) \subset \mathfrak{Z}$, Lemma 3.1 a) gives $\mathfrak{B} \cap U(\mathscr{G})^{\prime}=m(\mathfrak{Z})$. But, by Lemma 3.1d), $m(\mathfrak{B})^{-}=m\left(\mathfrak{Z}^{-}\right)=\mathfrak{B}^{-} \cap U(\mathscr{G})^{\prime}$. 
Note that if $\mathscr{G}$ acts on $\mathfrak{B}$, then $m(\mathfrak{B}) \subset \mathfrak{B}$ if either $\mathfrak{Z}$ is a von Neumann algebra or if $g \rightarrow \alpha_{g}(B)$ is a norm continuous mapping from $\mathscr{G}$ into $\mathfrak{B}$ for each $B \in \mathfrak{B} .^{6}$ We shall apply this Lemma to various subalgebras of $\mathfrak{F}$ and the crucial property which allows us to do so is that $m$ is local and normal as a mapping of $\mathfrak{F}$.

From the definition of the observable algebra in (1.9) and (1.10) we get

$$
\mathfrak{A}(\mathcal{O})=\mathfrak{F}(\mathcal{O}) \cap U(\mathscr{G})^{\prime}=m(\mathfrak{F}(\mathcal{O}))
$$

and by the norm continuity of $m$

$$
\mathfrak{A}=m(\mathfrak{F})=\mathfrak{F} \cap U(\mathscr{G})^{\prime} .
$$

Now $\mathfrak{F}$ is irreducible by assumption, so that

\section{by Lemma $3.2^{7}$.}

$$
\mathfrak{A}^{-}=m\left(\mathfrak{F}^{-}\right)=U(\mathscr{G})^{\prime}
$$

In view of Eq. (3.3), we may decompose the representation of $\mathfrak{A}$ by decomposing the representation of the gauge group. Let

$$
\begin{aligned}
\mathscr{H} & =\bigoplus_{\sigma \in \Sigma} \mathscr{H}_{\sigma} \otimes \mathscr{H}_{\sigma}^{\prime} \\
U(g) & =\bigoplus_{\sigma \in \Sigma} U_{\sigma}(g) \otimes I_{\sigma}^{\prime}
\end{aligned}
$$

be the factorial decomposition of the representation $U$ of the gauge group, with $\Sigma$ the set of equivalence classes of irreducible representations contained in $U, U_{\sigma}$ a representation of class $\sigma$ acting in the finite dimensional Hilbert space $\mathscr{H}_{\sigma} . \mathscr{H}_{\sigma}^{\prime}$ a Hilbert space of dimensionality equal to the multiplicity of $\sigma$, and $I_{\sigma}^{\prime}$ the unit operator on $\mathscr{H}_{\sigma}^{\prime}$. Let $E_{\sigma}$ denote the projection in $\mathscr{H}$ onto $\mathscr{H}_{\sigma} \otimes \mathscr{H}_{\sigma}^{\prime}$ then $E_{\sigma} \in U(\mathscr{G})^{\prime} \cap U(\mathscr{G})^{\prime \prime}=\mathscr{A}-\cap \mathfrak{A}^{\prime}$ by (3.3) so $\mathscr{H}_{\sigma} \otimes \mathscr{H}_{\sigma}^{\prime}$ reduces $\mathfrak{H}^{-} . A \in \mathfrak{A}$ acts on $\mathscr{H}_{\sigma} \otimes \mathscr{H}_{\sigma}^{\prime}$ as $I \otimes \pi_{\sigma}(A)$ where $\pi_{\sigma}$ is an irreducible representation of $\mathscr{A}$ in the Hilbert space $\mathscr{H}_{\sigma}^{\prime}$. $\pi_{\sigma}$ and $\pi_{\tau}$ are disjoint if $\sigma \neq \tau$ since their central supports $E_{\sigma}$ and $E_{\tau}$ are orthogonal [10; 5.2.1 (iii)]. Hence $\pi_{\sigma}$ and $\pi_{\tau}$ are inequivalent for $\sigma \neq \tau$.

The term "physical spectrum of $\mathfrak{A}$ " was introduced in section I to denote the equivalence classes of irreducible representations of $\mathfrak{A}$ occurring in our problem. Hence we have derived

3.3. Theorem. $\sigma \rightarrow \pi_{\sigma}$ defines a $1-1$ mapping from $\Sigma$ to the "physical spectrum" of $\mathfrak{A}$.

${ }^{6} \mathfrak{F}(\mathcal{O})$ is assumed to be a von Neumann algebra so this remark applies with $\mathfrak{B}=\mathfrak{F}(\mathcal{O})$ but also $g \rightarrow \alpha_{g}\left(F^{\prime}\right)$ cannot then, in general, be a norm continuous mapping from $\mathscr{G}$ into $\mathfrak{F}(\mathcal{O})$.

7 It is perhaps worth noting that the existence of a locally normal projection mapping $m: \mathfrak{F} \rightarrow \mathfrak{A}=\mathfrak{F} \cap U(\mathscr{G})^{\prime}$ in no way depends on the compactness of $\mathscr{G}$, since for local von Neumann algebras $\mathfrak{F}(\mathcal{O})$ we may apply the Theorem of Kovács and SzÜCS $[8,9]$ to construct such a mapping for an arbitrary group $\mathscr{G}$ acting locally and leaving $\Omega$ invariant. 
The purpose of the remainder of this section is to show that every irreducible representation of the gauge group must occur. The techniques will be those of [11] and involve constructing operators from the field algebra with definite tensor character. Given $\phi, \psi \in \mathscr{H}_{\sigma}$, we define, for $F \in \mathfrak{F}$

$$
M_{\phi, \psi}^{\sigma}(F)=\int_{\mathscr{G}}\left\langle\phi, U_{\sigma}(g) \psi\right\rangle \alpha_{g}(F) d \mu(g) .
$$

3.4. Lemma. Let $\sigma \in \Sigma$ and let $\iota \in \Sigma$ denote the trivial representation of $\mathscr{G}$. a) $F \rightarrow M_{\phi, \psi}^{\sigma}(F)$ is a norm-continuous linear mapping $\mathfrak{F} \rightarrow \mathfrak{F}$.

b) $\alpha_{g} \circ M_{\phi, \psi}^{\sigma}=M_{U_{\sigma}(g) \phi, \psi}^{\sigma}$.

c) $M_{\phi, \psi}^{\sigma}(F)\left(\mathscr{H}_{\iota} \otimes \mathscr{H}_{\iota}^{\prime}\right) \subset \mathscr{H}_{\sigma} \otimes \mathscr{H}_{\sigma}^{\prime}$.

d) If $\left\{\phi_{k}\right\}$ is an orthonormal basis for $\mathscr{H}_{\sigma}, \psi \in \mathscr{H}_{\sigma}, \psi \neq 0$ then there exists an $F \in \mathfrak{F}(\mathcal{O})$ such that $\left\{M_{\phi_{k}, \psi}^{\sigma}(F) \Omega\right\}$ is an orthonormal basis of a subspace of $\mathscr{H}$ carrying a representation equivalent to $\sigma$.

We omit the proof of this result which is of an elementary nature and which may be found in a different setting in [11; Theorem 4]. d) uses the cyclicity of $\Omega$ for $F(\mathcal{O})$ which is part of the Reeh-Schlieder theorem and shows in particular that $\mathfrak{F}(\mathcal{O})$ contains non-zero tensors of type $\sigma$.

3.5. Theorem. a) If $\sigma$ and $\sigma^{\prime} \in \Sigma$, there exists a subrepresentation of $U$ equivalent to $\sigma \otimes \sigma^{\prime}$. Hence $\sigma \otimes \sigma^{\prime}=\bigoplus_{i=1}^{n} \sigma_{i}$ with $\sigma_{i} \in \Sigma$.

b) If $\sigma \in \Sigma$, then $\bar{\sigma} \in \Sigma$.

Proof. a) Let $\sigma, \sigma^{\prime} \in \Sigma, \phi, \psi \in \mathscr{H}_{\sigma}, \phi^{\prime}, \psi^{\prime} \in \mathscr{H}_{\sigma^{\prime}}$, and $F, F^{\prime} \in \mathfrak{F}$. Then $\phi \otimes \phi^{\prime} \rightarrow M_{\phi, \psi}^{\sigma}(F) M_{\phi^{\prime}, \psi^{\prime}}^{\sigma^{\prime}}\left(F^{\prime}\right) \Omega$ defines a unique linear map $T$ from $\mathscr{H}_{\sigma} \otimes \mathscr{H}_{\sigma^{\prime}}$ onto a $\mathscr{G}$-invariant finite dimensional subspace $\mathscr{K}$ of $\mathscr{H}$. Further, by Lemma $3.4 \mathrm{~b}), T U_{\sigma}(g) \otimes U_{\sigma^{\prime}}(g)=U_{\mathscr{K}}(g) T$ where $U_{\mathscr{K}}$ is the restriction of $U$ to $\mathscr{K}$. If $T$ has a inverse, $U_{\sigma} \otimes U_{\sigma^{\prime}}$ and $U_{\mathscr{K}}$ are equivalent. This we show to be the case if $F$ and $F^{\prime}$ are suitably chosen. Now

$$
\left\langle T \phi_{1} \otimes \phi_{1}^{\prime}, T \phi_{2} \otimes \phi_{2}^{\prime}\right\rangle=\left\langle\Omega, M_{\phi_{1}^{\prime}, \psi^{\prime}}^{\sigma^{\prime}}\left(F^{\prime}\right) * M_{\phi_{1}, \psi}^{\sigma}(F) * M_{\phi_{2}, \psi}^{\sigma}(F) M_{\phi_{2}^{\prime}, \psi^{\prime}}^{\sigma^{\prime}}\left(F^{\prime}\right) \Omega\right\rangle .
$$

Choose $F \in \mathfrak{F}$ as in Lemma $3.4 \mathrm{~d})$ and $F^{\prime} \in \mathfrak{F}$ in the same way with $\sigma^{\prime}$ replacing $\sigma$. Replace $F$ by $\alpha_{\boldsymbol{x}}(F)$ and let the corresponding map $T$ be denoted by $T_{\boldsymbol{x}}$. Now $M_{\phi, \varphi}^{\boldsymbol{\sigma}}$ and $\alpha_{\boldsymbol{x}}$ commute so

$$
\begin{aligned}
& \left\langle T_{\boldsymbol{x}} \phi_{1} \otimes \phi_{1}^{\prime}, T_{\boldsymbol{x}} \phi_{2} \otimes \phi_{2}^{\prime}\right\rangle \\
& =\left\langle\Omega, M_{\phi_{1}^{\prime}, \psi^{\prime}}^{\sigma^{\prime}}\left(F^{\prime}\right) * \alpha_{\boldsymbol{x}}\left[M_{\phi_{1}, \psi}^{\sigma}(F) * M_{\phi_{2}, \psi}^{\sigma}\left(F^{\prime}\right)\right] M_{\phi_{2}^{\prime}, \psi^{\prime}}^{\sigma^{\prime}}\left(F^{\prime}\right) \Omega\right\rangle .
\end{aligned}
$$

But by the clustering property,

$$
\begin{aligned}
\lim _{|\boldsymbol{x}| \rightarrow \infty}\left\langle T_{\boldsymbol{x}} \phi_{1} \otimes \phi_{1}^{\prime}, T_{\boldsymbol{x}} \phi_{2} \otimes \phi_{2}^{\prime}\right\rangle \\
=\left\langle\Omega, M_{\phi_{1}, \psi}^{\mathrm{o}}(F) * M_{\phi_{2}, \psi}^{\sigma}(F) \Omega\right\rangle\left\langle\Omega, M_{\phi_{1}^{\prime}, \psi^{\prime}}^{\sigma^{\prime}}\left(F^{\prime}\right) * M_{\phi_{2}^{\prime}, \psi^{\prime}}^{\sigma^{\prime}}\left(F^{\prime}\right) \Omega\right\rangle \\
=\left\langle\phi_{1}, \phi_{2}\right\rangle\left\langle\phi_{1}^{\prime}, \phi_{2}^{\prime}\right\rangle .
\end{aligned}
$$


Thus $T_{\boldsymbol{x}}^{*} T_{\boldsymbol{x}} \rightarrow I$ as $|\boldsymbol{x}| \rightarrow \infty$ and in particular $T_{\boldsymbol{x}}$ has rauk $\operatorname{dim} \sigma \cdot \operatorname{dim} \sigma^{\prime}$ for $|\boldsymbol{x}|$ sufficiently large and hence has an inverse. This proves a).

b) Given $\sigma \in \Sigma, \phi, \psi \in \mathscr{H}_{\sigma}, \phi, \psi \neq 0$ we can by Lemma $\left.3.4 \mathrm{~d}\right)$ choose $F^{*} \in \mathfrak{F}(\mathcal{O})$ such that $M_{\phi, \psi}^{\sigma}\left(F^{*}\right) \neq 0$.

Then $M_{\phi, \psi}^{\sigma}\left(F^{*}\right)^{*}=\int_{\mathscr{G}} \overline{\left\langle\phi, U_{\sigma}(g) \psi\right\rangle} \alpha_{g}(F) d \mu(g) \neq 0, M_{\phi, \psi}^{\sigma}\left(F^{*}\right)^{*} \in \mathcal{F}(\mathcal{O})$.

But by the Reeh-Schlieder Theorem $\Omega$ is separating for $\mathfrak{F}(\mathcal{O})$ hence $M_{\phi, \varphi}^{\sigma}\left(F^{*}\right)^{*} \Omega$ is a non-zero vector transforming according to the representation $\bar{\sigma}$, so $\bar{\sigma} \in \Sigma$.

Recalling again the situation in Section II, where $\mathscr{G}$ can be identified with a subgroup of the internal symmetries of the single-particle multiplets, we can see why Theorem 3.5 should hold. It is an analogue of the Theorem on the additivity of the energy-momentum spectrum [12] and may be sharpened to include this result as well. Let $S_{\sigma}$ denote the energy-momentum spectrum in $\mathscr{H}_{\sigma}^{\prime}$ and let $p \in S_{\sigma}, p^{\prime} \in S_{\sigma^{\prime}}$. Then replacing $F$ and $F^{\prime}$ of Theorem 3.5 by $\int f(x) \alpha_{x}(F) d x$ and $\int f^{\prime}(x) \alpha_{x}\left(F^{\prime}\right) d x$ where supp $\tilde{f}$ and supp $\tilde{f}^{\prime}$ are contained in an arbitary neighbourhood of $p$ and $p^{\prime}$ respectively, we deduce that if $\sigma \otimes \sigma^{\prime}=\bigoplus_{i=1}^{n} \sigma_{i}$,

$$
S_{\sigma}+S_{\sigma^{\prime}} \subset S_{\sigma_{i}} \quad \text { for } \quad i=1,2, \ldots n .
$$

We can now show that every irreducible representation of the gauge group occurs.

3.6. Theorem. $\Sigma=\hat{\mathscr{G}}$, the complete spectrum of $\mathscr{G}$. Thus $\sigma \rightarrow \pi_{\sigma}$ defines a 1 - 1 mapping of $\hat{\mathscr{G}}$ onto the physical spectrum of $\mathfrak{A}$.

Proof. Let $\mathscr{S}$ denote the linear space of functions on $\mathscr{G}$ generated by the functions $f(g)=\left\langle\phi, U_{\sigma}(g) \psi\right\rangle$ with $\sigma \in \Sigma$ and $\phi, \psi \in \mathscr{H}_{\sigma}$. It suffices to show that $\mathscr{S}$ is dense in $L^{2}(\mathscr{G})$ since if $\sigma \notin \Sigma, \int \overline{f(g)}\left\langle\phi, U_{\sigma}(g) \psi\right\rangle$ $d \mu(g)=0$ for all $f \in \mathscr{S}$. Now $\mathscr{S}$ is a *-subalgebra of the $\mathrm{C}^{*}$-algebra of continuous functions on $\mathscr{G}, C(\mathscr{G})$, since it is clearly a linear subspace, and is closed under products and complex conjugation by Theorem 3.5. However $U$ is a faithful representation of $\mathscr{G}$, so $\mathscr{S}$ separates points of $\mathscr{G}$ and is norm dense in $C(\mathscr{G})$ by the Stone-Weierstrass theorem [13]. But $\mathscr{G}$ is compact, so $\mathscr{S}$ is also dense in $L^{2}(\mathscr{G})$.

This completes our analysis for it shows that the structure of that part of the spectrum of $\mathfrak{A}$ occurring in the decomposition of the given representation into irreducible components is determined by $\hat{\mathscr{G}}$, the spectrum of the gauge group. In particular, we have a multiplication law of sectors and a conjugation. Further if $\mathscr{G}$ is Abelian, $\hat{\mathscr{G}}$ is just the dual group of $\mathscr{G}$ and the family of sectors has the structure of a discrete Abelian group. 


\section{Duality}

We now investigate when the irreducible representation $\pi_{\sigma}$ of $\mathfrak{A}$ defined in the last section can be expected to satisfy duality [see Eq. (1.15)]. We shall assume first that (1.23) holds and postpone a discussion of its validity until after the proof of Theorem 4.1.

We call a sector $\sigma$ "Abelian" if $\operatorname{dim} \mathscr{H}_{\sigma}=1$ so that

$$
U(g) E_{\sigma}=E_{\sigma} U(g)=\chi_{\sigma}(g) E_{\sigma}
$$

where $\chi_{\sigma}$ is a character of $\mathscr{G}$. We then have

4.1. Theorem. If $\mathfrak{A}\left(\mathcal{O}^{\prime}\right)^{-}=m\left(\mathfrak{F}(\mathcal{O})^{\prime}\right)$ then $\mathfrak{A}$ satisfies duality for $\mathcal{O}$ in any Abelian sector.

Proof. Let $E$ be the central projection of $U(\mathscr{G})^{\prime}$ determining an Abelian sector and an irreducible representation $\pi$ of $\mathfrak{A}$.

$$
\pi(\mathfrak{A}(\mathcal{O}))^{\prime}=(\mathfrak{A}(\mathcal{O}) E)_{E \mathscr{H}}^{\prime}=\left(E \mathfrak{A}(\mathcal{O})^{\prime} E\right)_{E \mathscr{H}}
$$

by the von Neumann commutant Theorem [7; pp. 17-19]. Now

$$
E \mathfrak{A}(\mathcal{O})^{\prime} E=E \mathfrak{F}(\mathcal{O})^{\prime} \vee U(\mathscr{G})^{\prime \prime} E=E \mathfrak{F}(\mathcal{O})^{\prime} E \quad \text { by }(4.1) .
$$

Again (4.1) implies for any $T \in \mathscr{B}(\mathscr{H})$

Hence

$$
E m(T) E=E \int_{\mathscr{G}} U(g) T U(g)^{-1} d \mu(g) E=E T E .
$$

$$
E \mathfrak{A}(\mathcal{O})^{\prime} E=E m\left(\mathfrak{F}(\mathcal{O})^{\prime}\right) E
$$

and applying (1.23) we get

$$
E \mathfrak{A}(\mathcal{O})^{\prime} E=E \mathfrak{A}\left(\mathcal{O}^{\prime}\right)^{-} E .
$$

This yields the required statement of duality

$$
\pi(\mathfrak{A}(\mathcal{O}))^{\prime}=\pi\left(\mathfrak{A}\left(\mathcal{O}^{\prime}\right)\right)^{-} .
$$

In contrast to this result, we shall show in section $\mathrm{V}$ that duality does not hold in a non-Abelian sector. Actually under assumption 7, $(1.23)$ is a necessary condition for duality to hold for $\mathcal{O}$ in the vacuum sector.

We shall now consider a specialization of the commutation structure of $\mathfrak{F}$. This allows us to formulate a notion of duality for the field algebra itself from which the relation (1.23) follows. It will also provide a background for some of the discussion in the next section.

We consider von Neumann algebras on $\mathscr{H}$ stable under the action of the gauge group. We suppose that there is an operation on the set of such algebras called "twisting", $R \rightarrow R^{t}$ such that

$$
\begin{aligned}
& m\left(R^{t}\right)=m(R), \\
& m\left(R^{t^{\prime}}\right)=m\left(R^{\prime}\right) .
\end{aligned}
$$


4.2. Definition. $\mathscr{F}(\mathcal{O})^{t \prime}$ is called the "twisted commutant" of $\mathfrak{F}(\mathcal{O})$ and $\mathfrak{F}$ is said to satisfy "twisted locality" if

$$
\mathfrak{F}\left(\mathcal{O}^{\prime}\right)-\subset \mathfrak{F}(\mathcal{O})^{t^{\prime}} \quad \text { for each } \mathcal{O} \text {. }
$$

If $\mathfrak{F}$ is made up of Bose and Fermi fields, there is always a natural twisting structure such that $\mathfrak{F}$ satisfies twisted locality. In the case of normal commutation relations we construct it as follows. Let $\gamma \in \alpha(\mathscr{G})$ be the automorphism which changes the sign of Fermi fields and leaves Bose fields invariant. Then $F \in \mathcal{F}$ may be written $F=F_{+}+F_{-}$where $F_{+}=1 / 2(F+\gamma(F))$ and $F_{-}=1 / 2(F-\gamma(F))$. Further $m(F)=m\left(F_{+}\right)$. Let $U$ implement $\gamma$, then if $R$ is invariant under $\mathscr{G}$ (invariant under $\gamma$ would do) we define

$$
R^{t}=\left\{F_{+}+U F_{-}: F \in R\right\} .
$$

Clearly $m\left(R^{t}\right)=m(R)$ which is (4.4) and in this case we have the further properties $R^{t t}=R$ and $R^{t^{\prime}}=R^{\prime t}$. Hence $m\left(R^{t^{\prime}}\right)=m\left(R^{\prime t}\right)=m\left(R^{\prime}\right)$ which gives (4.5). For the free Fermi field we can take $U=e^{i \pi Q}=e^{i \pi N}$, where $Q$ is the charge operator and $N$ the particle number operator. $R^{t}$ is then obtained from $R$ by applying a Klein transformation. There are also more complicated examples of twisting structure when $\mathfrak{F}$ is made up of Bose and Fermi fields but with abnormal commutation relations where different fields do not necessarily either commute or anticommute with each other.

4.3. Definition. $\mathfrak{F}$ is said to satisfy "twisted duality" for $\mathcal{O}$ if

$$
\mathfrak{F}\left(\mathcal{O}^{\prime}\right)^{-}=\mathfrak{F}(\mathcal{O})^{t^{\prime}}
$$

Of course duality is the special case of twisted duality where $R^{t}=R$. We show in the Appendix that the free Fermi field satisfies twisted duality for any open double cone $\mathcal{O}$. When $\mathfrak{F}$ satisfies twisted duality the relation (1.23) is an immediate consequence since

$$
m\left(\mathfrak{F}(\mathcal{O})^{\prime}\right)=m\left(\mathfrak{F}(\mathcal{O})^{t^{\prime}}\right)=m\left(\mathfrak{F}\left(\mathcal{O}^{\prime}\right)^{-}\right)=\mathfrak{A}\left(\mathcal{O}^{\prime}\right)^{-}
$$

where the last step uses the fact that $m$ is local and normal, see Lemma 3.2.

\section{Relative Commutants and Weak Duality}

5.1. Definition. A representation $\pi$ of $\mathfrak{A}$ is said to satisfy "weak duality" for $\mathcal{O}$ if

$$
\pi\left(\mathfrak{A}^{c}(\mathcal{O})\right)^{-}=\pi(\mathfrak{A}(\mathcal{O}))^{\prime} \cap \pi(\mathfrak{A})^{-}
$$

where the relative commutant $\mathfrak{A}^{c}(\mathcal{O})$ is defined by (1.19).

If (5.1) holds for the defining representation of the concrete $C^{*}$. algebra $\mathfrak{A}$ on $\mathscr{H}$, we simply say that $\mathfrak{A}$ itself satisfies weak duality for $\mathcal{O}$. The crucial difference between weak duality and duality is that $\mathfrak{A}^{c}(\mathcal{O})$ takes the place of $\mathfrak{A}\left(\mathcal{O}^{\prime}\right)$. The intersection with $\pi(\mathfrak{A})$ - is taken on 
the right hand side of (5.1) only because the representation $\pi$ is not necessarily irreducible. Since $\mathfrak{A}\left(\mathcal{O}^{\prime}\right) \subset \mathfrak{A}^{c}(\mathcal{O})$, duality in the representation $\pi$ implies weak duality in that representation.

5.2. Theorem. If $\mathfrak{A}$ satisfies weak duality for $\mathcal{O}$, i.e. if $\mathfrak{A}^{c}(\mathcal{O})^{-}=\mathfrak{A}(\mathcal{O})^{\prime}$ $\cap \mathfrak{A}^{-}$then $\pi_{\sigma} \mid \mathfrak{I}^{c}(\mathcal{O})$ and $\pi_{\sigma^{\prime}} \mid \mathfrak{A}^{c}(\mathcal{O})$ are disjoint for $\sigma \neq \sigma^{\prime}$ and weak duality holds for $\mathcal{O}$ in each representation $\pi_{\sigma}$.

Proof. If $E$ is a central projection of $\mathfrak{A}^{\prime}$, then $E \in \mathfrak{A}(\mathcal{O})^{\prime} \cap \mathfrak{A}^{-}=\mathfrak{A}^{c}(\mathcal{O})^{-}$ so $E$ is also a central projection of $\mathfrak{A}^{c}(\mathcal{O})^{-}$. But $E_{\sigma}$ and $E_{\sigma^{\prime}}$ are orthogonal for $\sigma \neq \sigma^{\prime}$, so the representations of $\mathfrak{A}^{c}(\mathcal{O})$ in the spaces $E_{\sigma} \mathscr{H}$ and $E_{\sigma^{\prime}} \mathscr{H}$ are disjoint $[10 ; 5.2 .1$ (iii)]. Further weak duality holds in the subrepresentation on $E \mathscr{H}$ :

we have

$E \mathfrak{A}^{c}(\mathcal{O})^{-}=E\left\{\mathfrak{U}(\mathcal{O})^{\prime} \cap \mathfrak{A}^{-}\right\} E \subset E \mathfrak{A}(\mathcal{O})^{\prime} E \cap \mathfrak{A}^{-} E \subset \mathfrak{A}(\mathcal{O})^{\prime} \cap \mathfrak{A}^{-}=\mathfrak{A}^{c}(\mathcal{O})^{-}$

where the first inclusion is obvious and the second one holds because $E$ is in $\mathfrak{A}(\mathcal{O})^{\prime}$ and $\mathfrak{A}-$. Hence

$$
E \mathfrak{A}^{c}(\mathcal{O})^{-}=E \mathfrak{A}(\mathcal{O})^{\prime} E \cap \mathfrak{A}^{-} E
$$

Applying von Neumann's theorem [7; pp. 17-19] we get

$$
E \mathfrak{A}^{c}(\mathcal{O})^{-}=\left\{E \mathfrak{A}^{c}(\mathcal{O})\right\}^{-}=\{E \mathfrak{A}(\mathcal{O})\}^{\prime} \cap\{E \mathfrak{A}\}^{-}
$$

and our assertion holds. In particular for $E=E_{\sigma}$,

$$
E_{\sigma} \mathfrak{A}^{-}=I \otimes \mathscr{B}\left(\mathscr{H}_{\sigma}^{\prime}\right)=\left\{\mathscr{B}\left(\mathscr{H}_{\sigma}\right) \otimes I\right\}^{\prime} \quad \text { by }[7 ; \text { p. 26, Proposition 4] }
$$

and using the same Proposition again we get

$$
\begin{aligned}
I \otimes \pi_{\sigma}\left(\mathfrak{A}^{c}(\mathcal{O})\right)^{-} & =\left\{I \otimes \pi_{\sigma}(\mathfrak{U}(\mathcal{O}))\right\}^{\prime} \cap\left\{\mathscr{B}\left(\mathscr{H}_{\sigma}\right) \otimes I\right\}^{\prime} \\
& =\left\{\mathscr{B}\left(\mathscr{H}_{\sigma}\right) \otimes \pi_{\sigma}(\mathfrak{U}(\mathcal{O}))\right\}^{\prime}=I \otimes \pi_{\sigma}(\mathfrak{A}(\mathcal{O}))^{\prime} .
\end{aligned}
$$

Thus $\pi_{\sigma}\left(\mathfrak{A}^{c}(\mathcal{O})\right)^{-}=\pi_{\sigma}(\mathfrak{A}(\mathcal{O}))^{\prime}$ which is the required statement of weak duality in the representation $\pi_{\sigma}$.

We want to show next that weak duality in the defining representation of $\mathfrak{A}$ follows from two assumptions on $\mathfrak{F}$. The first assumption, "weak twisted duality" for $\mathfrak{F}$, is defined by analogy with (5.1) using the "relative twisted commutant":

$$
\mathfrak{F}^{t c}(\mathcal{O})=\mathfrak{F}(\mathcal{O})^{t^{\prime}} \cap \mathfrak{F}
$$

$\mathfrak{F}$ is said to satisfy weak twisted duality for $\mathcal{O}$ if

$$
\mathfrak{F}^{t c}(\mathcal{O})^{-}=\mathfrak{F}(\mathcal{O})^{t^{\prime}}
$$

The second assumption is on the gauge automorphisms:

Assumption 8 . Given any $\mathcal{O}$ and any $g \in \mathscr{G}$ there is a unitary operator $V_{g} \in \mathfrak{F}$ (depending on $\mathcal{O}$ ) such that

$$
\alpha_{g}(F)=V_{g} F V_{g}^{-1} \quad \text { for all } \quad F \in \mathfrak{F}(\mathcal{O})^{t}
$$


We expect this to be valid when $U(g)$ may be derived from a local density. In the case of the free charged scalar field discussed in this context in the introduction after Eq. (1.20) we would set

$$
V_{0}=\exp \left[i \theta j_{0}\left(f_{R} g_{\delta}\right)\right], 0 \leqq \theta<2 \pi .
$$

We may rewrite $(5.4)$ as $V_{g}^{-1} U(g) \in \mathfrak{F}(\mathcal{O})^{t^{\prime}}$; the twisted commutant appears here rather than the commutant since, roughly speaking, we expect $V_{g}^{-1} U(g) \in \mathfrak{F}\left(\mathcal{O}^{\prime}\right)$ - and under the assumption of twisted locality, $\mathfrak{F}\left(\mathcal{O}^{\prime}\right)-\subset \mathfrak{F}(\mathcal{O})^{t^{\prime}}$. Note that in the standard cases where $\mathfrak{F}$ has Bose-Fermi structure and $V_{g}, U(g)$ commute with the Klein operator, one also has $V_{g}^{-1} U(g) \in \mathfrak{F}(\mathcal{O})^{\prime}$ which means that the gauge automorphisms on $\mathfrak{F}(\mathcal{O})$ can be implemented by unitary elements from $\mathfrak{F}$.

We now show that if $\mathfrak{F}$ satisfies weak twisted duality for $\mathcal{O}$ and assumption 8 holds, then $\mathfrak{A}$ satisfies weak duality for $\mathcal{O}$. In other words we have:

5.3. Theorem. If $\mathfrak{F}^{t c}(\mathcal{O})^{-}=\mathfrak{F}(\mathcal{O})^{t^{\prime}}$, and if for each $g \in \mathscr{G}$ there is a unitary operator $V_{g} \in \mathfrak{F}$ such that $V_{g}^{-1} U(g) \in \mathfrak{F}(\mathcal{O})^{t^{\prime}}$, then $\mathfrak{A}^{c}(\mathcal{O})^{-}=\mathfrak{A}(\mathcal{O})^{\prime} \cap \mathfrak{A}$.

Proof. $\mathfrak{H}^{c}(\mathcal{O})=\mathfrak{A}(\mathcal{O})^{\prime} \cap \mathfrak{A}=m\left(\mathfrak{A}(\mathcal{O})^{\prime} \cap \mathfrak{F}\right)$. Hence as $m$ is local and normal $\mathfrak{A}^{c}(\mathcal{O})^{-}=m\left(\left\{\mathfrak{A}(\mathcal{O})^{\prime} \cap \mathfrak{F}\right\}^{-}\right)$. Thus it suffices to show that

$$
\left\{\mathfrak{U}(\mathcal{O})^{\prime} \cap \mathfrak{F}\right\}^{-}=\mathfrak{A}(\mathcal{O})^{\prime} .
$$

Now $\mathfrak{F}^{t c}(\mathcal{O})^{-} \subset\left\{\mathfrak{A}(\mathcal{O})^{\prime} \cap \mathfrak{F}\right\}-$ so, by hypothesis,

$$
\mathfrak{F}(\mathcal{O})^{t^{\prime}} \subset\left\{\mathfrak{A}(\mathcal{O})^{\prime} \cap \mathfrak{F}\right\}^{-} \text {. }
$$

But $V_{g} \in \mathfrak{A}(\mathcal{O})^{\prime} \cap \mathfrak{F}$ and $V_{g}^{-1} U(g) \in \mathfrak{F}(\mathcal{O})^{t^{\prime}}$, hence $U(g) \in\left\{\mathfrak{U}(\mathcal{O})^{\prime} \cap \mathfrak{F}\right\}$ But this holds for all $g \in \mathscr{G}$ so

$$
U(\mathscr{G})^{\prime \prime} \subset\left\{\mathfrak{A}(\mathcal{O})^{\prime} \cap \mathfrak{F}\right\}^{-} .
$$

Now by $(4.4) \mathfrak{A}(\mathcal{O})=m\left(\mathfrak{F}(\mathcal{O})^{t}\right)$ so that $\mathfrak{A}(\mathcal{O})^{\prime}=\mathfrak{F}(\mathcal{O})^{t^{\prime}} \vee U(\mathscr{G})^{\prime \prime}$ and (5.6) and (5.7) give $\mathfrak{A}(\mathcal{O})^{\prime} \subset\left\{\mathfrak{U}(\mathcal{O})^{\prime} \cap \mathfrak{F}\right\}-$. However the reverse inequality is trivial, so we have (5.5) and with it the theorem.

Weak duality (5.1) was first assumed by Borchers [2; R 8] for the irreducible representations $\pi$ describing individual sectors; it was motivated by the statement [2; Lemma III - 1] that the representations of $\mathfrak{A}^{c}(\mathcal{O})$ in the different sectors are unitarily equivalent. Weak duality in the sectors would then imply that the various intertwining operators between these representations differ from one another by observables from $\mathfrak{A}(\mathcal{O})$, and thus admit an interpretation as local fields. However theorems 5.2 and 5.3 show that the representations of $\mathfrak{A}^{c}(\mathcal{O})$ in the different sectors are disjoint in typical cases so that intertwining operators do not exist. We show in the next section that the representations of $\mathfrak{A}\left(\mathcal{O}^{\prime}\right)$ in the different sectors are unitarily equivalent. Weak duality is not a strong enough property to allow the intertwining operators between these representations to be interpreted as fields. However that interpre- 
tation is again possible under the stronger assumption of duality in each sector.

In Section IV we showed how relation (1.23) implies duality for $\mathcal{O}$ in each Abelian sector. We now show that, under our assumptions, we can never get duality in non-Abelian sectors. We begin with a Lemma characterizing Abelian sectors.

5.4 Lemma. $\sigma \in \hat{\mathscr{G}}$ gives rise to an Abelian sector if and only if $\sigma \subset \sigma \otimes \tau, \tau \in \hat{\mathscr{G}}$, implies that $\tau=\iota$, the identity representation.

Proof. We note first of all that if $\varrho, \tau \in \hat{\mathscr{G}}$, then $\iota \subset \varrho \otimes \tau$ if and only if $\varrho=\tilde{\tau}$ and $\iota$ is contained just once in $\bar{\tau} \otimes \tau$. This follows at once from the orthogonality relations for the characters of a compact group [14; $\S 32]$. Suppose now $\operatorname{dim} \sigma=1$ and $\sigma \subset \sigma \otimes \tau$. Then $\bar{\sigma} \otimes \sigma=\imath$ since $\operatorname{dim} \bar{\sigma} \otimes \sigma=\operatorname{dim} \sigma^{2}=1$. Hence $\iota=\bar{\sigma} \otimes \sigma C \bar{\sigma} \otimes(\sigma \otimes \tau)=\iota \otimes \tau=\tau$ so $\iota=\tau$. Conversely suppose $\operatorname{dim} \sigma>1$, then $\bar{\sigma} \otimes \sigma \supset \varrho$ with $\varrho \neq \imath$. Now $\bar{\sigma} \otimes(\varrho \otimes \sigma)$ $=\bar{\varrho} \otimes(\bar{\sigma} \otimes \sigma) \supset \bar{\varrho} \otimes \varrho \supset \iota$. Hence $\bar{\varrho} \otimes \sigma \supset \sigma$ and we may take $\tau=\bar{\varrho}$.

5.5. Lemma. If $\mathfrak{A}$ satisfies weak duality for $\mathcal{O}$ and $F \in \mathfrak{F}(\mathcal{O})$ is a nonzero tensor of character $\tau \neq \iota$ then there exists an $A \in \mathfrak{A}^{c}(\mathcal{O})$ such that $(F A-A F) \Omega \neq 0$.

Proof. As in the proof of Theorem 5.2, a central projection of $\mathfrak{A}^{\prime}$ is a central projection of $\mathfrak{A}^{c}(\mathcal{O})^{-}$. Hence $E_{\iota} \in \mathfrak{Q}^{c}(\mathcal{O})^{-}$. But $\left(F E_{\iota}-E_{\iota} F\right) \Omega$ $=F \Omega \neq 0$ by the Reeh-Schlieder Theorem. Hence since we can approximate $E_{\imath}$ weakly by elements of $\mathfrak{A}^{c}(\mathcal{O})$, there exists an $A \in \mathfrak{A}^{c}(\mathcal{O})$ with $(F A-A F) \Omega \neq 0$.

Note that Theorem 5.2 already tells us that $\mathfrak{F}(\mathcal{O})$ cannot commute with $\mathfrak{A}^{c}(\mathcal{O})$ because otherwise a tensor of character $\tau$ from $\mathfrak{F}(\mathcal{O})$ would be an intertwining operator between the representations of $\mathfrak{A}^{c}(\mathcal{O})$ in $E_{\tau} \mathscr{H}$ and $E_{\iota} \mathscr{H}$. On the other hand assumption 7 states that $\mathfrak{F}(\mathcal{O})$ commutes with $\mathfrak{A}\left(\mathcal{O}^{\prime}\right)$. Hence

$$
\mathfrak{A}\left(\mathcal{O}^{\prime}\right)^{-} \neq \mathfrak{A}^{c}(\mathcal{O})^{-} .
$$

If we do not make use of any of the "weak duality" properties then assumption 7 leads by the same reasoning to

$$
\mathfrak{A}\left(\mathcal{O}^{\prime}\right)^{-} \neq \mathfrak{A}(\mathcal{O})^{\prime} \cap \mathfrak{A}^{-} .
$$

5.6. Theorem. If $\mathfrak{A}$ satisfies weak duality for $\mathcal{O}$, then duality does not hold for $\mathcal{O}$ in any non-Abelian sector.

Proof. Suppose $\sigma \in \hat{\mathscr{G}}$ defines a non-Abelian sector, then by Lemma 5.4 there exists a $\tau \in \hat{\mathscr{G}}, \tau \neq \iota$ such that $\sigma \subset \sigma \otimes \tau$. By Lemma 3.4d), $\mathscr{F}(\mathcal{O})$ contains a non-zero tensor $F_{\tau}$ of character $\tau$. By Lemma 5.5 there exists an $A \in \mathfrak{A}^{c}(\mathcal{O})$ such that $C_{\tau} \Omega \equiv\left(A F_{\tau}-F_{\tau} A\right) \Omega \neq 0$. Let $\mathrm{C}_{\tau}^{i}, i=1,2 \ldots$ $\operatorname{dim} \tau$ be a basis of the tensor multiplet of character $\tau$ to which $C_{\tau}$ belongs. Then using the clustering property as in Theorem 3.5, we can choose 2 Commun.math.Phys.,Vol.13 
a basis $F_{\sigma}^{j}, j=1,2, \ldots, \operatorname{dim} \sigma$ of a tensor multiplet of character $\sigma$ so that $C_{\tau}^{i} F_{\sigma}^{j} \Omega$ forms a basis of vectors of the representation $\sigma \otimes \tau$. But $\sigma \subset \sigma \otimes \tau$ so that $E_{\sigma} C_{\tau} E_{\sigma} \neq 0$. Hence

$$
E_{\sigma}\left(A F_{\tau}-F_{\tau} A\right) E_{\sigma} \neq 0 .
$$

We now show that $\pi_{\sigma}(A) \notin \pi_{\sigma}\left(\mathfrak{A}\left(\mathcal{O}^{\prime}\right)\right)$-. For if it were, there would exist a $B \in \mathfrak{A}\left(\mathcal{O}^{\prime}\right)^{-}$such that $E_{\sigma} A=E_{\sigma} B$. But $B \in \mathfrak{F}(\mathcal{O})^{\prime}$ by assumption 7 . Hence

$$
0=E_{\sigma}\left(B F_{\tau}-F_{\tau} B\right) E_{\sigma}=E_{\sigma}\left(A F_{\tau}-F_{\tau} A\right) E_{\sigma}
$$

contradicting (5.9). Hence $\pi_{\sigma}(A) \in \pi_{\sigma}\left(\mathfrak{A}^{c}(\mathcal{O})\right) \subset \pi_{\sigma}(\mathfrak{A}(\mathcal{O}))^{\prime}$ but $\pi_{\sigma}(A) \notin$ $\pi_{\sigma}\left(\mathcal{H}\left(\mathcal{O}^{\prime}\right)\right)^{-}$. In other words duality does not hold for $\mathcal{O}$ in the sector $\pi_{\sigma}$.

We may note that if instead of the inequality sign in (5.8) we had an equality sign, duality would hold in every sector in contrast to Theorem 5.6. Furthermore one could conclude that the representations of $\mathfrak{A}\left(\mathcal{O}^{\prime}\right)$ in distinct sectors were disjoint, whereas we shall show in the next section that they are unitarily equivalent.

\section{Strong Local Equivalence and Localized Automorphisms}

The first aim in this section is to prove that the representations of $\mathfrak{A}$ in the different sectors are strongly locally equivalent. Here we must use assumption 7 that the observables commute with space-like separated fields and the Reeh-Schlieder property, assumption 6 .

Since we do not assume any specific commutation properties of the fields with themselves, (1.18) does not necessarily follow from (1.17). However assumption 7 goes some way towards justifying (1.18); in fact, (1.20) implies

$$
\bigcap_{x \in \boldsymbol{R}^{4}} \mathfrak{F}(\mathcal{O}+x) \subset \mathfrak{A}^{\prime}=U(\mathscr{G})^{\prime \prime}
$$

and if we also assume

then

$$
\mathfrak{F}_{\cap} U(\mathscr{G})^{\prime \prime}=\{\lambda I\}
$$

$$
\bigcap_{x \in \boldsymbol{R}^{4}} \mathfrak{F}(\mathcal{O}+x)=\{\lambda I\} .
$$

Eq. (1.18) follows from (6.3) by taking commutants. The additional assumption (6.2) allows the physical interpretation that the gauge transformations are not quasilocal quantities.

Now let $\pi_{1}$ and $\pi_{2}$ be any two subrepresentations of $\mathfrak{A}$ (in particular, two sectors), then as a first step we show that the restrictions of $\pi_{1}$, and $\pi_{2}$ to $\mathfrak{A}\left(\mathcal{O}^{\prime}\right)$ are quasiequivalent for any $\mathcal{O}^{\prime}$, i.e. that

$$
\pi_{1}\left|\mathfrak{A}\left(\mathcal{O}^{\prime}\right) \approx \pi_{2}\right| \mathfrak{A}\left(\mathcal{O}^{\prime}\right) .
$$

It is clearly sufficient to prove this when $\pi_{1}$ is the defining representation of $\mathfrak{A}$. Let $E \in \mathfrak{A}^{\prime}$ be the projection such that $\pi_{2}$ is the restriction of $\pi_{1}$ 
to $E \mathscr{H}$ :

$$
\pi_{2}(A)=A E \quad \text { on } \quad E \mathscr{H} .
$$

Since $\mathfrak{A}^{\prime} \subset \mathfrak{A}\left(\mathcal{O}^{\prime}\right)^{\prime}, E \in \mathfrak{A}\left(\mathcal{O}^{\prime}\right)^{\prime}$. Now by $[10 ; 5.3 .1(\mathrm{v})],(6.4)$ holds if and only if the central support $E^{\prime}$ of $E$ in $\mathfrak{A}\left(\mathcal{O}^{\prime}\right)^{\prime}$ is equal to I. However

$$
E^{\prime}=\left[\mathfrak{A}\left(\mathcal{O}^{\prime}\right)^{\prime} \vee \mathfrak{A}\left(\mathcal{O}^{\prime}\right)-E \mathscr{H}\right]=\left[\mathfrak{A}\left(\mathcal{O}^{\prime}\right)^{\prime} E \mathscr{H}\right]^{8} .
$$

Further since $E \in \mathfrak{A}^{\prime}=U(\mathscr{G})^{\prime \prime} \subset U(\mathscr{P} \uparrow)^{\prime}$, we can find an a.f.e.-vector $\Psi \in E \mathscr{H}$. Thus from (1.20) and the Reeh-Schlieder property

$$
E^{\prime} \geqq[\mathfrak{F}(\mathcal{O}) E \mathscr{H}] \geqq[\mathfrak{F}(\mathcal{O}) \Psi]=I
$$

Hence $E^{\prime}=I$ and we have established (6.4).

Now the quasiequivalence in (6.4) implies unitary equivalence if $\pi_{j}\left(\mathfrak{U}\left(\mathcal{O}^{\prime}\right)\right)^{-}, j=1,2$ both have cyclic and separating vectors $[7 ;$ p. 233 Thm. 3]. However a.f.e.-vectors are cyclic for $\mathfrak{F}(\mathcal{O})$ by assumption 6 and hence separating for $\mathfrak{A}\left(\mathcal{O}^{\prime}\right)$ from (1.20). Further $\bigcup_{x \in \mathbf{R}^{4}} \mathfrak{A}\left(\mathcal{O}^{\prime}+x\right)$ is norm dense in $\mathfrak{A}$ so that for any representation $\pi$

$$
\underset{x \in \boldsymbol{R}^{4}}{\bigvee} \pi\left(\mathfrak{A}\left(\mathcal{O}^{\prime}+x\right)\right)=\pi(\mathfrak{A})^{-} \text {. }
$$

Hence by the Reeh-Schlieder Theorem [4] a.f.e.-vectors will be cyclic for $\pi_{j}\left(\mathfrak{A}\left(\mathcal{O}^{\prime}\right)\right)^{-}$if and only if they are cyclic for $\pi_{j}(\mathfrak{U})^{-}$. This is always true for irreducible representations. Moreover, if there are a countable number of sectors, i.e. if $\mathscr{G}$ is separable, $\mathfrak{A}$ itself and hence any $\pi_{j}$ has cyclic a.f.e.-vectors. In particular we have now proved

6.1. Theorem. Let $\pi_{0}, \pi_{\sigma}$ be irreducible subrepresentations of $\mathfrak{A}$ corresponding to the vacuum sector and the sector $\sigma, \sigma \in \hat{\mathscr{G}}$, acting on subspaces $\mathscr{H}_{0}^{\prime}$ and $\mathscr{H}_{\sigma}^{\prime}$ of $\mathscr{H}$. Then there is a unitary operator $V_{\sigma}: \mathscr{H}_{0}^{\prime} \rightarrow \mathscr{H}_{\sigma}^{\prime}$ such that

$$
V_{\sigma} \pi_{0}(A)=\pi_{\sigma}(A) V_{\sigma}, A \in \mathfrak{A}\left(\mathcal{O}^{\prime}\right) .
$$

In general, nothing more can be said about these intertwining operators $V_{\sigma}$; however under the assumptions of the next Theorem, they can be related to special elements of $\mathfrak{F}(\mathcal{O})$.

6.2. Theorem. If $\sigma$ is Abelian and if $m\left(\mathfrak{F}(\mathcal{O})^{\prime}\right)=\mathfrak{A}\left(\mathcal{O}^{\prime}\right)^{-}$, then given a unitary operator $V_{\sigma}$ satisfying $(6.9)$, there is a unitary operator $\psi_{\sigma} \in \mathfrak{F}(\mathcal{O})$ of tensor character $\sigma$, such that

$$
V_{\sigma}=E_{\sigma} \psi_{\sigma} E_{0} \quad \text { on } \quad E_{0} \mathscr{H} .
$$

Proof. As $\Omega$ is cyclic and separating for $\mathfrak{F}(\mathcal{O})^{\prime}$ by assumption 6 , we can introduce a densely defined linear operator $\psi_{\sigma}$ on $\mathscr{H}$ such that

$$
\psi_{\sigma} F \Omega=F V_{\sigma} \Omega \quad \text { if } \quad F \in \mathscr{F}(\mathcal{O})^{\prime},
$$

${ }^{8}$ We use square brackets to denote the projection onto the closure of a linear subspace. 
where $\mathscr{H}_{\sigma}^{\prime}$ is identified with $E_{\sigma} \mathscr{H}$ and hence $V_{\sigma} \Omega \in \mathscr{H}$. We verify that $\psi_{\sigma}$ can be extended to a unitary operator on $\mathscr{H}$. Now

$$
E_{0} \mathfrak{F}(\mathcal{O})^{\prime} \Omega=m\left(\mathfrak{F}(\mathcal{O})^{\prime}\right) \Omega=\mathfrak{A}\left(\mathcal{O}^{\prime}\right)^{-} \Omega .
$$

However (6.9), (6.11) and (6.12) show that $\psi_{\sigma}$ coincides with $V_{\sigma}$ on $E_{0} \mathfrak{F}(\mathcal{O})^{\prime} \Omega$ which is dense in $E_{0} \mathscr{H}=\mathscr{H}_{0}^{\prime}$. If $F \in \mathscr{F}(\mathcal{O})^{\prime}, m\left(F^{*} F\right) \in m\left(\mathfrak{F}(\mathcal{O})^{\prime}\right)$ $=\mathfrak{A}\left(\mathcal{O}^{\prime}\right)^{-}$; furthermore since $\sigma$ is Abelian, (4.1) implies that $E_{\sigma} F^{*} F E_{\sigma}$ $=m\left(F^{*} F\right) E_{\sigma}$. Hence by $(6.9)$

$$
\left\|\psi_{\sigma} F \Omega\right\|^{2}=\left(V_{\sigma} \Omega, F^{*} F V_{\sigma} \Omega\right)=\|F \Omega\|^{2} .
$$

Since $\left[\mathfrak{F}(\mathcal{O})^{\prime} \Omega\right]=I$, this means that $\psi_{\sigma}$ can be extended to map $\mathscr{H}_{\sigma^{\prime}}^{\prime}$ isometrically into $\mathscr{H}_{\sigma \sigma^{\prime}}^{\prime}$ for each $\sigma^{\prime} \in \hat{\mathscr{G}}$. Hence $\psi_{\sigma}$ becomes isometric on $\mathscr{H},(6.10)$ is satisfied and by $(6.11)$

$$
\psi_{\sigma} \in \mathfrak{F}(\mathcal{O})^{\prime \prime}=\mathfrak{F}(\mathcal{O}) \text {. }
$$

It remains to show that $\psi_{\sigma}$ is unitary. Now since $V_{\sigma}$ is unitary we may pick $\Phi \in \mathscr{H}_{0}^{\prime}$ such that $V_{\sigma} \Phi=\psi_{\sigma} \Phi$ is an a.f.e.-vector. Hence by $(6.14)$ and assumption 6

$$
\left[\psi_{\sigma} \mathfrak{F}(\mathcal{O})^{\prime} \Phi\right]=\left[\mathfrak{F}(\mathcal{O})^{\prime} \psi_{\sigma} \Phi\right]=I
$$

and $\psi_{\sigma}$ maps onto $\mathscr{H}$.

It is important to note that when $\mathscr{G}$ is Abelian $\psi^{*} A \psi$ is an observable for each $A \in \mathfrak{A}$ and each $\psi \in \mathfrak{F}$ of definite tensor character. Combining this remark with what precedes we derive

6.3. Corollary. Under the assumptions of Theorem 6.2, there is an automorphism $\gamma_{\sigma}$ of $\mathfrak{A}$ for each Abelian $\sigma \in \hat{\mathscr{G}}$ such that

$$
\pi_{\sigma} \cong \pi_{0} \circ \gamma_{\sigma}
$$

and a unitary operator $\psi_{\sigma} \in \mathfrak{F}(\mathcal{O})$ of tensor character $\sigma$ implementing $\gamma_{\sigma}$

$$
\gamma_{\sigma}(A)=\psi_{\sigma}^{*} A \psi_{\sigma}, \quad A \in \mathfrak{A} .
$$

We therefore see that in the case of an Abelian gauge group and a field algebra satisfying (1.23) the sectors of $\mathfrak{A}$ are connected to one another by automorphisms of $\mathfrak{A}$ acting trivially on $\mathfrak{A}\left(\mathcal{O}^{\prime}\right)$ and leaving the set $\mathfrak{A}(\mathcal{O})$ invariant. We call such automorphisms "automorphisms localized in $\mathcal{O}^{\prime}$. Notice that such an automorphism also leaves the set $\mathfrak{Z}^{c}(\mathcal{O})$ invariant but it must, from the results of the preceding section, induce a non-trivial automorphism of $\mathfrak{A}^{c}(\mathcal{O})$.

The existence of localized automorphisms connecting the different sectors is characteristic of an Abelian gauge group. We shall show in a second paper how the group of localized automorphisms of a given algebra of observables describes an Abelian group of superselection quantum numbers carried by Bose or Fermi fields. Theorems 4.2 and 5.6 show the intimate connexion between an Abelian gauge group and duality for 
the observable algebra in every sector. The following Proposition shows directly how the existence of localized automorphisms is governed by duality in the sectors.

6.4. Proposition. Let $\mathcal{O}_{1}$ and $\mathcal{O}_{2}$ be double cones such that $\mathcal{O}_{1}$ contains the closure of $\mathcal{O}_{2}$. Suppose further that $\mathfrak{A}$ is generated by $\mathfrak{A}\left(\mathcal{O}_{1}\right)$ and $\mathfrak{A}\left(\mathcal{O}_{2}^{\prime}\right)$ and that $\pi_{0}$ and $\pi_{\sigma}$ are two irreducible representations of $\mathfrak{A}$ such that $\pi_{0}(\mathfrak{A})$ has the duality property

$$
\pi_{0}\left(\mathfrak{Z}\left(\mathcal{O}_{1}\right)\right)=\pi_{0}\left(\mathfrak{Z}\left(\mathcal{O}_{1}^{\prime}\right)\right)^{\prime}
$$

and that the restrictions of $\pi_{0}$ and $\pi_{\sigma}$ to $\mathfrak{A}\left(\mathcal{O}_{2}\right)$ are equivalent as in (6.9). Then there is an automorphism of $\mathfrak{A}$ localized in $\mathcal{O}_{1}$ and connecting $\pi_{0}$ to $\pi_{\sigma}$, i.e. satisfying $(6.16)$, if and only if $\pi_{\sigma}(\mathfrak{H})$ also enjoys the duality property $(6.18)$.

Proof. The "only if" part is trivial. Assume $\pi_{\sigma}(\mathfrak{U})$ satisfies (6.18). By (6.9) the restrictions of $\pi_{0}$ and $\pi_{\sigma}$ to $\mathfrak{A}\left(\mathcal{O}_{1}^{\prime}\right)$ are unitarily equivalent hence $V_{o} \pi_{0}\left(\mathfrak{A}\left(\mathcal{O}_{1}^{\prime}\right)\right) V_{\sigma}^{-1}=\pi_{\sigma}\left(\mathfrak{A}\left(\mathcal{O}_{1}^{\prime}\right)\right)$. Taking commutants and using $(6.18)$ for $\pi_{0}(\mathfrak{U})$ and $\pi_{\sigma}(\mathfrak{A})$

$$
V_{\sigma} \pi_{0}\left(\mathfrak{A}\left(\mathcal{O}_{1}\right)\right) V_{\sigma}^{-1}=\pi_{\sigma}\left(\mathfrak{A}\left(\mathcal{O}_{1}\right)\right) .
$$

But $\mathfrak{A}\left(\mathcal{O}_{1}\right)$ and $\mathfrak{A}\left(\mathcal{O}_{2}^{\prime}\right)$ generate $\mathfrak{A}$. Therefore (6.9) for $\mathcal{O}_{2}^{\prime}$ and $(6.19)$ imply that $V_{\sigma} \pi_{0}(\mathfrak{U}) V_{\sigma}^{-1}=\pi_{\sigma}(\mathfrak{Q})$. Hence

$$
V_{\sigma}^{-1} \pi_{\sigma}(A) V_{\sigma}=\pi_{0}\left(\gamma_{\sigma}(A)\right)
$$

defines a localized automorphism $A \rightarrow \gamma_{\sigma}(A)$ with the desired properties.

A state $\omega$ of $\mathfrak{Z}$ is "strictly localized" [15] if it is equal to the vacuum state on some $\mathfrak{A}\left(\mathcal{O}^{\prime}\right)$. The characteristic assumption of this section, assumption 7 , has the consequence that each sector contains a total set of strictly localized states.

\section{Appendix}

\section{Twisted Duality for the Free Fermi Field}

Consider a free field of half-integral spin and mass $m \geqq 0$, and let $\mathcal{O}$ be a bounded open double cone and $\mathcal{O}^{\prime}$ its space-like complement. It follows from the results of DeLL'Antonio [16] that the Hilbert space $\mathscr{H}$ of the Fock representation can be represented as an incomplete infinite tensor product with respect to the vector $\Omega=\bigotimes_{i} \Omega_{i}$ :

$$
\mathscr{H}=\bigotimes_{i}^{\Omega}\left(\mathscr{H}_{i} \otimes \mathscr{H}_{i}^{\prime}\right),
$$

where $\mathscr{H}_{i}$ and $\mathscr{H}_{i}^{\prime}$ are two-dimensional Hilbert spaces, in such a way that

1. There exist operators $A_{i}, B_{i} \in \mathscr{B}(\mathscr{H}), i=1,2 \ldots$ satisfying the canonical anticommutation relations (CAR) with $A_{i} \Omega=B_{i} \Omega=0$. (Note that $\Omega \in \mathscr{H}$ is not the Fock vacuum). 
2. There exist operators $\alpha_{i}, \beta_{i} \in \mathscr{B}(\mathscr{H}), i=1,2 \ldots$ satisfying the CAR such that

$$
\begin{aligned}
\alpha_{i} & =c_{i} A_{i}-s_{i} B_{i}^{*} \\
c_{i}^{2}+s_{i}^{2} & =1 \\
\beta_{i} & =s_{i} A_{i}^{*}+c_{i} B_{i}
\end{aligned}
$$

and $\mathfrak{F}(\mathcal{O})$ and $\mathfrak{F}\left(\mathcal{O}^{\prime}\right)^{-}$are the von Neumann algebras generated by $\left\{\alpha_{i}: i=1,2 \ldots\right\}$ and $\left\{\beta_{i}: i=1,2 \ldots\right\}$ respectively.

3. $\alpha_{i}=P_{1} \otimes P_{2} \otimes \cdots \otimes P_{i-1} \otimes\left[\left(\begin{array}{ll}0 & 1 \\ 0 & 0\end{array}\right) \otimes I_{i}^{\prime}\right] \otimes I \otimes I \otimes \cdots$ where $P_{j}$ denotes the matrix $\left(\begin{array}{rr}1 & 0 \\ 0 & -1\end{array}\right) \otimes I^{\prime}$ in the $j^{\text {th }}$ place.

$$
\beta_{i}=P_{1} \otimes P_{2} \otimes \cdots \otimes P_{i-1} \otimes\left[\left(\begin{array}{rr}
1 & 0 \\
0 & -1
\end{array}\right) \otimes\left(\begin{array}{ll}
0 & 1 \\
0 & 0
\end{array}\right)\right] \otimes R_{i+1} \otimes R_{i+2} \otimes \cdots
$$

where $R_{j}$ denotes the matrix $\left(\begin{array}{rr}1 & 0 \\ 0 & -1\end{array}\right) \otimes\left(\begin{array}{rr}1 & 0 \\ 0 & -1\end{array}\right)$ in the $j^{t h}$ place. $\Omega_{i}=c_{i}|0\rangle_{\alpha_{i}}|0\rangle_{\beta_{i}}$ $-s_{i}|1\rangle_{\alpha_{i}}|1\rangle_{\beta_{i}}$, using an obvious notation.

Now, see (4.7), $\mathfrak{F}\left(\mathcal{O}^{\prime}\right)^{-t}$ is generated by $\left\{e^{i \pi N} \beta_{i}: i=1,2 \ldots\right\}$ where $N$ is the total particle number operator. Further

$$
e^{i \pi N} \beta_{i}=Q_{1} \otimes Q_{2} \otimes \cdots \otimes Q_{i-1} \otimes\left[I_{i} \otimes\left(\begin{array}{ll}
0 & 1 \\
0 & 0
\end{array}\right)\right] \otimes I \otimes I \otimes \cdots
$$

where $Q_{j}$ denotes the matrix $I \otimes\left(\begin{array}{rr}1 & 0 \\ 0 & -1\end{array}\right)$ in the $j^{\text {th }}$ place. Now let $M_{i}$ denote the factor $\mathscr{B}\left(\mathscr{H}_{i}\right) \otimes I_{i}^{\prime}$, then $M_{i}^{\prime}=I_{i} \otimes \mathscr{B}\left(\mathscr{H}_{i}^{\prime}\right)$ and $\mathscr{F}(\mathcal{O})=\bigotimes_{i}^{\Omega} M_{i}$, $\mathfrak{F}\left(\mathcal{O}^{\prime}\right)^{-t}=\bigotimes_{i}^{\Omega} M_{i}^{\prime}$. But by [17; Lemma 6.10] $\left(\bigotimes_{i}^{\Omega} M_{i}\right)^{\prime}=\bigotimes_{i}^{\Omega} M_{i}^{\prime}$. Hence $\mathfrak{F}\left(\mathcal{O}^{\prime}\right)^{-t}=\mathfrak{F}(\mathcal{O})^{\prime}$ so that $\mathfrak{F}\left(\mathcal{O}^{\prime}\right)^{-}=\mathfrak{F}(\mathcal{O})^{\prime t}=\mathfrak{F}(\mathcal{O})^{t^{\prime}}$ which is the required statement of twisted duality.

Acknowledgements. S. D. gratefully acknowledges the hospitality of the Deutsches Elektronen-Synchrotron during the final stages of this collaboration. J.E.R. wishes to thank The Royal Society for a research fellowship under their European Programme.

Note added in proof. The following result expresses the connexion between localized automorphisms and duality in the sectors more conveniently than Proposition 6.4.

Let $\mathcal{O}_{1}$ be a double cone and suppose that the vacuum representation $\pi_{0}$ has the duality property

$$
\pi_{0}(\mathfrak{A}(\mathcal{O}))=\pi_{0}\left(\mathfrak{A}\left(\mathcal{O}^{\prime}\right)\right)^{\prime}
$$

for each double cone $\mathcal{O} \supset \mathcal{O}_{1}$. If $\pi_{\sigma}$ is a representation of $\mathfrak{A}$ unitarily equivalent to $\pi_{0}$ when restricted to $\mathfrak{A}\left(\mathcal{O}_{1}^{\prime}\right)$ then there is an automorphism of $\mathfrak{A}$ localized in $\mathcal{O}_{1}$ and connecting $\pi_{0}$ to $\pi_{\sigma}$, i.e. satisfying (6.16), if and only if $\pi_{\sigma}(\mathfrak{Q})$ also enjoys the duality property $(6.21)$. 
This result is proved as in Proposition 6.4 noting that (6.19) now holds for all double cones $\mathcal{O} \supset \mathcal{O}_{1}$.

Theorem 3.6 for the case of an Abelian gauge group is essentially contained in JADCZYK [18].

\section{References}

1. HAAG, R., and D. Kastler: An algebraic approach to quantum field theory. J. Math. Phys. 5, 848-861 (1964).

2. Borchers, H. J.: Local rings and the connection of spin with statistics. Commun. Math. Phys. 1, 281-307 (1965).

3. Borchers, H. J.: The vacuum state in quantum field theory II. Commun. Math. Phys. 1, 57-79 (1965).

4. Borchers, H. J.: On the converse of the Reeh-Schlieder theorem. Commun. Math. Phys. 10, 269-273 (1968).

5. Araki, H., and R. HAAG: Collision cross sections in terms of local observables. Commun. Math. Phys. 4, 77-91 (1967).

6. Bourbaki, N.: Topologie générale, Ch I, 3rd. ed. Paris: Hermann 1961.

7. Dixmier, J.: Les algèbres d'opérateurs dans l'espace hilbertien. Paris: GauthierVillars 1957.

8. Kovács, I., and J. Szücs: Ergodic type theorems in von Neumann algebras. Acta Sc. Math. 27, 233-246 (1966).

9. Doplicher, S., D. Kastler, and E. Størmer: Invariant states and asymptotic Abelianness. To appear J. Funct. Analysis.

10. Dixmier, J.: Les C*-algèbres et leurs répresentations. Paris: Gauthier-Villars 1964.

11. Doplicher, S., and D. Kastler: Ergodic states in a non-commutative ergodic theory. Commun. Math. Phys. 7, 1-20 (1968).

12. WightMAN, A. S.: Recent achievements of axiomatic field theory in "Theoretical Physics" IAEA, Vienna (1963).

13. Stone, M. H.: Applications of the theory of Boolean rings to general topology. Trans. Am. Math. Soc. 41, 375-481 (1937).

14. Nammark, M. A.: Normed rings. Groningen: P. Noordhoff N. V. 1959.

15. KNIght, J. M.: Strict localization in quantum field theory. J. Math. Phys. 2, $459-471(1961)$.

16. Dell'Antonio, G. F.: Structure of the algebras of some free systems. Commun. Math. Phys. 9, 81-117 (1968).

17. Araki, H., and E. J. Woods: A classification of factors. Publ. RIMS, Kyoto Univ. Ser. A3, 51-130 (1968).

18. JADCZYK, A. Z.: On the spectrum of internal symmetries in algebraic quantum field theory. Commun. Math. Phys. 12, 58-63 (1969).

S. DOPLICHER

CNRS, Physique Théorique

31, Ch. J. Aiguier

F 13 Marseille
R. HAAG

J. E. RoBERTS

II. Institut f. Theoret. Physik

der Universität

2000 Hamburg 50, Luruper Chaussee 149 